\section{Behavioral reflections of the NMDA system}

\section{URSULA STAUBLI \\ University of California, Irvine, California}

LTP is formed quickly, persists for very long periods of time, and is synapse-specific-properties which suggest that it might be involved in certain types of memory. Rapid induction and persistence point to learning that $o c-$ curs in a few trials and that is retained without rehearsal for a very long time. The synapse specificity of LTP suggests an association with a high-capacity memory system, since theoretical considerations imply that the selectivity feature is necessary for the encoding of large amounts of information. Starting with these ideas, we used two types of drugs known to block LTP and tested them in a learning paradigm that involves the rapid acquisition of many cues of a similar type. Specifically, we examined the effects of a protease inhibitor (calpain) and an NMDAreceptor antagonist (APV) in a task in which rats were required to learn a long series of two-odor discriminations, with each discrimination being acquired by experienced animals in 5-10 trials. This learning paradigm also has the feature that the rats retain the discriminations for very long periods. Thus, the characteristics of the tested memory (i.e., rapid acquisition, many items, and stable encoding) correspond somewhat to those expected of an LTP-based system, and we found that drugs that block LTP block rapid encoding. This is not to say that the animals never learned the problems, but rather that they were no longer able to learn them rapidly (Staubli, Baudry, \& Lynch, 1985; Staubli, Thibault, DiLorenzo, \& Lynch, 1989).

It will be noted that the NMDA receptor, which seems to be obligatory for induction of LTP, is anatomically restricted in its distribution; it is found in high concentrations in the outer layers of cortex, some pathways of the hippocampus, and other regions of the telencephalon, but it is not present in high density in any number of pathways at various levels of the brain (Monaghan \& Cotman, 1985). Thus, the assumption can be made that there will be forms of memory available to the animal that do not involve LTP. It follows that drugs that block LTP via NMDA-receptor antagonism should be useful in defining types of memories, not simply in the sense of behavioral paradigms but also in terms of neurobiological underpinnings. Thus, we find it interesting that the animals are capable of learning olfactory problems even in the presence of NMDA antagonists or other drugs that block LTP, given that enough trials are available to them. This

Correspondence may be addressed to Ursula Staubli, Center for the Neurobiology of Leaming and Memory, University of California, Irvine, CA 92717 suggests to us that there is a second type of memory system, a non-NMDA-receptor-dependent form of memory that the animals can fall back upon. Presumably this learning system is qualitatively different from the NMDAreceptor-based memory system in some sense other than being slower, but this is a matter for future research. These ideas should not be surprising, since hippocampal lesions block the rapid acquisition of two-odor discriminations, but do not block learning if the rats are given a sufficiently large number of trials (Staubli, Fraser, Kessler, \& Lynch, 1986).

There are other clues indicating that animals have more than one system available to them to learn even so simple a task as a two-cue discrimination. The deficit in the rapid acquisition just noted is found when two odors are presented simultaneously, but not if they are delivered sequentially (Eichenbaum, Fagan, Mathews, \& Cohen, 1988; Otto, Schottler, Staubli, Eichenbaum, \& Lynch, in press).

This brings us to the comments by Keith and Rudy in the present issue of Psychobiology, which, we feel, say more about their preconceptions regarding fundamental forms of memory than anything about the data in the papers under discussion. It will be noted that what we suggest as being an LTP-based memory is not widely studied by animal psychologists. Learning experiments with rats do not typically involve problems in which the animal is asked to encode dozens of cues of similar types, nor is there a heavy premium placed on rapid acquisition as opposed to learning over many trials. Ironically enough, these features are implicit in most studies done on human memory; word lists, photographs, and so forth are problems that involve the sampling and rapid encoding of an extensive series of similar cues. Keith and Rudy seem to think that we did not block memory encoding in our study, since the drug interfered only with rapid learning and did not prevent the ultimate acquisition of the discriminations. Our preconceptions, beginning with the neurobiological properties of LTP, are quite different and are noted above. Moreover, the several pathways leading out of the olfactory cortex (which itself has several subdivisions) lead us to assume that several types of processing are going on in parallel, some of which involve the NMDA receptors and some of which do not. In brief, what we see in Keith and Rudy's complaint are two sets of preconceptions coming into collision: our notions about the properties of LTP and the anatomy of the olfactory system, as opposed to Keith and Rudy's expectations, which are based on behavioral experiments with rats and on the history of animal psychology. What seems to be a substantial impairment from one perspective apparently is not seen as such from the other. We are, however, reasonably confident that human beings showing the type of failure of rapid acquisition that we have observed in our animal studies would be judged as having a severe memory impairment. 
The second point that Keith and Rudy raise is that we have not "convincingly" ruled out sensorimotor impairments as an explanation for our results. Sensorimotor processing is a variable that cannot be defined, let alone quantified, and thus it can never be ruled out. The same can be said for other constructs such as arousal, attention, motivation, and so forth. This is one reason why no pharmacological study is by itself going to provide conclusive evidence that any neurobiological process is critically involved in the actual plasticity or encoding stage of memory. The pharmacological results are one of a set of lines of evidence that hopefully will converge on a single answer regarding the possible role of NMDA receptors and LTP in memory. For example, it has been shown that LTP appears in the synapses involved in encoding or processing of information as the animal is learning that information (Roman, Staubli, \& Lynch, 1987). Thus, there is correlational as well as manipulative evidence. It has also been found that physiological activity patterns that occur in the hippocampus during learning are ideally suited for producing the LTP effect (Larson \& Lynch, 1986). It is misleading of Keith and Rudy to pretend that we or any others have claimed that pharmacological work alone is sufficient to evaluate the role of LTP in memory. As is clearly stated in the papers, this evidence goes into the same evaluative bin as results from studies of the type just noted.

This is not to say that variables only indirectly involved in synaptic plasticity can be ignored in pharmacological studies of the type under discussion. It is our position that the best that one can hope to do about regulating these variables is to set up an experiment in which all features are the same, except that in one instance learning is required and in another instance it is not. We attempted to do this by having the animals perform exactly the same task, using cues that had already been learned as opposed to ones that had not been learned. Given that performance to already learned cues was normal in the presence of the drug, we have difficulty accepting as a first explanation that the animals were somehow impaired in motivation or sensory properties, and so forth.

We would be more inclined to take Keith and Rudy's criticism more seriously if they had identified a few studies in which sensorimotor impairments had been "convincingly" controlled. This would have made it relatively easy for us to demonstrate that variables which cannot be accurately measured cannot be conclusively excluded.

To summarize, we would agree that pharmacological experiments are useful, even necessary, in the studies of the biology of memory, but that, by themselves, they cannot be sufficient. This is all too true, and all too familiar. With regard to the issue of controls, we think that our studies have introduced a paradigm that should be valuable for future work, a paradigm that involves familiar as opposed to novel cues of the same type tested in the same behavioral task within the same session. Drugs that interfere with the behavior when novel information is presented, but have no effect when familiar information is being processed, are good candidates for agents that affect the encoding process. Finally, the pharmacological studies that we and others have carried out suggest distinctions in memory types that may not align with those familiar to rat psychologists. Some will see this as an opportunity to explore the nature of memory; others will find it a cause for complaint.

\section{REFERENCES}

Eichenbaum, H., Fagan, A., Mathews, P., \& Cohen, N. (1988). Hippocampal system dysfunction and odor discrimination: Impairment and facilitation depending on cognitive strategies. Behavioral Neuroscience, 102, 331-339.

LARSON, J., \& LYNCH, G. (1986). Induction of synaptic potentiation in hippocampus by patterned stimulation involves two events. Science, 232, 985-988.

Monaghan, D. T., \& Cotman, C. W. (1985). Distribution of N-methylD-aspartate sensitive $\mathrm{L}-[3 \mathrm{H}]$-glutamate binding sites in rat brain. Journal of Neuroscience, 11, 2909-2919.

Otto, T., Schottler, F., Staubli, U., Eichenbaum, H., \& L,YNCH, G. (in press). The hippocampus and olfactory learning: Effects of entorhinal cortex lesions on learning-set acquisition and on odor memory in a successive-cue go, no-go task. Behavioral Neuroscience.

Roman, F., Staubli, U., \& LYNCH, G. (1987). Evidence for synaptic potentiation in a cortical network during learning. Brain Research, 418, 221-226.

Staubli, U., Baudry, M., \& LYNCh, G. (1985). Olfactory discrimination leaming is blocked by leupeptin, a thiol protease inhibitor. Brain Research, 337, 333-336.

Staubl, U., Fraser, D., Kessler, M., \& LyNCH, G. (1986). Studies on retrograde and anterograde amnesia of olfactory memory after denervation of the hippocampus by entorhinal cortex lesions. Behavioral \& Neural Biology, 46, 432-444.

Staubli, U., Thibault, O., Dilorenzo, M., \& Lynch, G. (1989). Antagonism of NMDA receptors impairs acquisition but not retention of olfactory memory. Behavioral Neuroscience, 103, 54-60. 